3 Phoa K, von Vilstern, F. Radiofrequency Ablation in Barrett's esophagus with confirmed low-Grade dysplaisa: interm results of a European multipcentre randomized controlled trial (SURF). Gastroenterology 2013: 5 Supplement1 S187

Disclosure of Interest None Declared.

\section{PTU-156 HEPATOCYTE NUCLEAR FACTOR 4 ALPHA (HNF4A) IS DEMONSTRATED IN BARRETT'S METAPLASIA, BUT NOT IN NORMAL HUMAN OESOPHAGUS}

1,2LP Griffiths*, 'Z Burke, ${ }^{2} \mathrm{JM}$ Farrant, ${ }^{2} \mathrm{~B}$ Colleypriest, 'D Tosh. ${ }^{1}$ Biology and Biochemistry, University of Bath, UK; ${ }^{2}$ Gastroenterology, Royal United Hospital Bath, Bath, UK

\subsection{6/gutjnl-2014-307263.230}

Introduction Barrett's metaplasia (BM) is the main risk factor for oesophageal adenocarcinoma, a cancer which carries a mortality of $>50 \%$ at 12 months. Refluxate containing gastric and bile acids seems to be causative for inflammation at the lower oesophagus, but it is not known how this induces replacement of stratified squamous epithelium (SSQE) with columnar epithelium at a molecular level.

There is likely to be a progenitor cell population replacing denuded epithelium, although the origin of these cells has not been proven. Genes that play a role in gut tissue patterning during embryogenesis have received attention. One such 'master switch' our laboratory is investigating encodes the hepatocyte nuclear factor 4 alpha $(\mathrm{HNF} 4 \alpha)$ transcription factor.

Methods We optimised an immunohistochemistry protocol for demonstrating HFN4 $\alpha$ on formalin-fixed paraffin-embedded slides of human tissue. This protocol was applied to forceps biopsy specimens of normal oesophagus, gastro-oesophageal junction (GOJ), stomach, ileum, colon and BM (UK REC reference: 13/YH/0197). Tissues were examined from at least 3 different patients per anatomical site.

Results In healthy tissues, nuclear HNF $4 \alpha$ positive immunostaining was demonstrated in stomach, ileum and colonic epithelium, but not in normal SSQE in the oesophagus. At the GOJ, there was clear delineation between $\mathrm{HNF} 4 \alpha$ positive nuclei in the columnar gastric cardia mucosa, and negative $\mathrm{HNF} 4 \alpha$ staining of SSQE. In contrast, the columnar epithelial nuclei in BM were consistently positive.

Conclusion HNF4 $\alpha$ transcription factor is demonstrable in BM, but not SSQE. We are not aware that this $\mathrm{HNF} 4 \alpha$ gastrointestinal distribution has been previously published. HNF $4 \alpha$ is likely to be a key transcription factor in the pathogenesis of BM.

Previous work in our laboratory with a mouse explant tissue culture model has shown that another candidate transcription factor responsible for $\mathrm{BM}(\mathrm{Cdx} 2)$ was insufficient to induce an intestinal phenotype, whereas $\mathrm{HNF} 4 \alpha$ induced villin, K18, trefoil factor 3 and mucin 5AC. We propose a 2-hit hypothesis for the development of BM:

1. induction of HNF4 $\alpha$ (which initially converts the oesophageal SSQE to columnar epithelium) and

2. Cdx2 (which causes intestinalisation of the columnar epithelium).

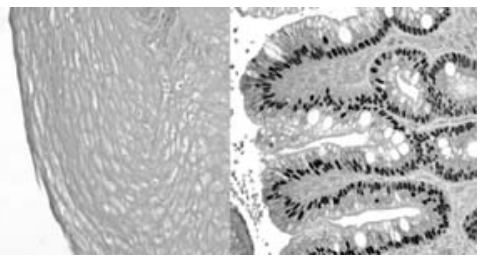

Abstract PTU-156 Figure 1
Demonstration of $\mathrm{HNF} 4 \alpha$ in BM but not SSQE is supportive of this theory.

Disclosure of Interest None Declared.

\section{PTU-157 OUTCOMES OF OESOPHAGEAL DILATATION IN ACHALASIA AND POST-FUNDOPLICATION DYSPHAGIA}

${ }^{1} \mathrm{M}$ Kasi ${ }^{*},{ }^{1} S$ Ahmad, ${ }^{2} \mathrm{~J}$ Wright, ${ }^{3} \mathrm{~K}$ Knowles, ${ }^{1} \mathrm{M}$ Fox. ${ }^{1}$ Gastroenterology, NHS, Nottingham, UK; ${ }^{2}$ GI Surgery, NHS, Nottingham, UK; ${ }^{3}$ GI Physiology, NHS, Nottingham, UK

10.1136/gutjnl-2014-307263.231

Introduction Dilatation of the Oesophago-Gastric Junction (OGJ) provides effective symptom relief in $58-95 \%$ of patients with achalasia, similar to that achieved by Heller's myotomy. Dilatation is also used in patients with persistent ( $>6$ months) dysphagia after fundoplication surgery; there is insufficient safety and outcome data of this procedure. Our aim is to compare patient outcome of endoscopic dilatation for both these conditions.

Methods We present 18 month experience of referrals to the dysphagia service 2012-2013. All patients underwent a diagnostic gastroscopy with biopsies, excluding inflammation or neoplasia. Patients with achalasia or clinically relevant outlet obstruction post-fundoplication diagnosed by elevated integrated relaxation pressure $(>25 \mathrm{mmHg})$ on high resolution manometry were selected. Dilatation was performed by 30 $35 \mathrm{~mm}$ Rigiflex II Balloon or Savary-Gillard Bougies (max $18 \mathrm{~mm}$ ) under fluoroscopic guidance. Primary outcome was symptom response at 3-6 months post-procedure by clinic or telephone follow-up. Overall symptom response was documented on an analogue scale from $0 \%>100 \%$ (inadequate $<40 \%$, satisfactory $40-60 \%$, good $60-80 \%$ and excellent $>80 \%)$.

Results 46/71 referrals had either achalasia or dysphagia post fundoplication. $30(41 \%)$ had achalasia, 6 had prior Heller's myotomy and 7 had prior Botulinum toxin. 16 (22\%) patients had OGJ obstruction after fundoplication. 29/30 patients with achalasia underwent pneumatic dilatation, one bougie dilatation. Overall symptom response was inadequate in $5(16 \%$ referred for surgery), satisfactory in $3(11 \%)$ and good-excellent in 22 (73\%).14/16 patients with post fundoplication dysphagia had pneumatic balloon dilatation, 2 had bougie dilatation. Overall symptom response was inadequate in 7 (44\% referred for surgery), satisfactory in 4 (25\%) and goodexcellent in $5(31 \%)$. Complications from the both groups include chest pain $(\mathrm{n}=2)$, chest infection $(\mathrm{n}=1)$, reflux symptoms ( $\mathrm{n}=4$ in each group) and minor bleeding. All resolved with conservative treatment.

More than half of achalasia and post-fundoplication patients reported "at least satisfactory" outcome 3-6 months after dilatation (84\% vs. 56\%; p < 0.07 Fisher Exact Test). A good-excellent symptom response was reported more often by achalasia patients $(\mathrm{p}=0.010)$.

Conclusion Endoscopic dilatation is safe and effective treatment for patients with dysphagia related to achalasia and also OGJ obstruction post-fundoplication. A good-excellent response was reported less frequently by the post-fundoplication patients; however more than half had at least "satisfactory" symptom relief and, therefore, a trial of endoscopic dilatation can be considered a viable alternative to reoperation.

Disclosure of Interest None Declared. 